\title{
The Possibility of Many Compensation Points in a Mixed-Spin Ising Ferrimagnetic System
}

\author{
Hadey K. Mohamad ${ }^{1,2}$ \\ ${ }^{1}$ College of Science, Al-Muthanna University, Samawa, Iraq \\ ${ }^{2}$ Voronezh State University, Universitetskaya pl. 1, Voronezh 394006, Russia \\ Correspondence should be addressed to Hadey K. Mohamad; hadeyk2002@yahoo.com
}

Received 4 April 2013; Accepted 9 May 2013

Academic Editors: V. Kochereshko, S. Krukowski, R. Rossmanith, and S. Wang

Copyright (c) 2013 Hadey K. Mohamad. This is an open access article distributed under the Creative Commons Attribution License, which permits unrestricted use, distribution, and reproduction in any medium, provided the original work is properly cited.

\begin{abstract}
The magnetic properties of a ferrimagnetic mixed spin-3/2 and spin-5/2 Ising model with different anisotropies are investigated by using the mean-field approximation (MFA). In particular, the effect of magnetic anisotropies on the compensation phenomenon, acting on $A$-atoms and $B$-ones for the mixed-spin model, has been considered in a zero field. The free energy of a mixed-spin Ising ferrimagnetic system from MFA of the Hamiltonian is calculated. By minimizing the free energy, we obtain the equilibrium magnetizations and the compensation points. The phase diagram of the system in the anisotropy dependence of transition temperature has been discussed as well. Our results of this model predict the existence of many (two or three) compensation points in the ordered system on a simple cubic lattice.
\end{abstract}

\section{Introduction}

Recently, there has been great interest in stable crystalline room-temperature magnets with spontaneous moments because of their potential device application in thermomagnetic recording, electronic and computer technologies. Besides that, ferrimagnetic ordering plays a crucial role in several novel materials based on molecular compounds $[1$, $2]$. Thus, the study of the Ising model with mixed spins of different magnitudes has attracted considerable attention. Important advance has been directed to the two-sublattice mixed-spin systems with single-ion anisotropies. These systems have been investigated by a variety of techniques, such as exact, mean-field approximation, and effective field theory [3-6]. For example, the authors [3] have investigated one constituent having spin-1 and the other constituent having spin-3/2. It has been shown that an outstanding result of this system was discovered experimentally in $\left(\mathrm{Ni}_{0.22}^{\mathrm{II}} \mathrm{Mn}_{0.60}^{\mathrm{II}} \mathrm{Fe}_{0.18}^{\mathrm{II}}\right)_{1.5}\left[\mathrm{Cr}^{\mathrm{III}}(\mathrm{CN})_{6}\right] \cdot 7 \cdot 6 \mathrm{H}_{2} \mathrm{O}[3]$. The purpose of this work is to investigate a more general mixed-spin Ising model consisting of spin-3/2 and spin-5/2. Our theoretical work may be classified into two types. In the first type, the spin compensation temperature of the system $\left(T_{k}\right)$ can be obtained by requiring the total magnetization to be equal to zero for various values of anisotropies though the reduced magnetization of the sublattices forming the system is not equal to zero [3,7-10]. The second class of work is the phase transitions which demand the Landau expansion of the free energy in the order parameter $[3,8,11]$. However, we firstly determine the ground-state phase diagram and study sublattice magnetizations of the mixed-spin ferrimagnetic Ising system with various values of the anisotropies on the basis of the mean-field theory. The basic framework of the theory based on the Bogoliubov inequality for the free energy has been presented. The phase diagrams and sublattice magnetizations for various values of the single-ion anisotropies acting on both atoms have been discussed.

\section{Theory}

We start by summarizing analytical results obtained for the MFA. The central idea of the mean-field theory is very simple: we treat the nearest neighbour Ising model in zero field on a lattice containing two sublattices $A, B$ having $N$ sites, each site 
having $z$ nearest neighbour. The Hamiltonian of the system in a zero field, with $\sigma_{i}^{A}=3 / 2$ and $\sigma_{j}^{B}=5 / 2$, can be written as follows $[3,9]$ :

$$
H=-\sum_{i, j} J_{i j} \sigma_{i}^{A} \sigma_{j}^{B}-D_{A} \sum_{i}\left(\sigma_{i}^{A}\right)^{2}-D_{B} \sum_{j}\left(\sigma_{j}^{B}\right)^{2}
$$

where the sites of sublattice $A$ are occupied by spins $\sigma_{i}^{A}$ taking the values of $\pm 1 / 2, \pm 3 / 2$, and the sites of sublattice $B$ are occupied by spins $\sigma_{j}^{B}$ taking the values of $\pm 1 / 2, \pm 3 / 2$, and $\pm 5 / 2 . D_{A}, D_{B}$ are the anisotropies acting on the spin-3/2 and spin-5/2, respectively. $J_{i j}$ is the exchange interaction between spins at sites $i$ and $j$. The expectation value of the spin variable at the site $i$ or $j$ is given by [10]

$$
\left\langle\sigma_{i / j}\right\rangle=\frac{1}{Z} \sum_{\left\{\sigma_{i}, \sigma_{j}\right\}} \sigma_{i / j} e^{-\beta H}
$$

with

$$
Z=\sum_{\left\{\sigma_{i} \sigma_{j}\right\}} e^{-\beta H}
$$

where $Z$ is the partition function of the system and $\sum$ means the sum over allowed states of the system. Here, $\beta=1 / K_{B} T$.

A systematic way of deriving the mean-field theory for a given microscopic Hamiltonian is to start from the Bogoliubov inequality $[3,11]$ :

$$
G \leq \Phi=G_{o}+\left\langle H-H_{o}\right\rangle_{o}
$$

where $G$ is the free energy of the system, $H_{o}$ a trial Hamiltonian depending on variational parameters, $G_{0}$ the corresponding free energy, and $\langle\cdots\rangle_{o}$ denotes an average taken in the ensemble defined by $H_{o}$.

The mean-field free energy is then defined by minimizing $\Phi$ with respect to the variational parameters $\lambda_{A / B}$, such that:

$$
G_{m f}=\min _{\lambda_{A / B}}\{\Phi\} .
$$

This gives the best possible approximation to the true free energy for a given choice of $H_{o}$, since inequality (4) insists that the mean-field free energy cannot fall below the true free energy. In this work we consider one of the simplest possible choices of $H_{o}$ :

$$
H_{o}=-\sum_{i}\left[\lambda_{A} \sigma_{i}^{A}+D_{A}\left(\sigma_{i}^{A}\right)^{2}\right]-\sum_{j}\left[\lambda_{B} \sigma_{j}^{B}+D_{B}\left(\sigma_{j}^{B}\right)^{2}\right],
$$

where $\lambda_{A}$ and $\lambda_{B}$ are the two variational parameters related to the two different spins, respectively. Then, the approximated free energy can be obtained by substituting the Hamiltonian of the system in (2); that is one has

$$
\begin{gathered}
g \equiv \frac{\Phi}{N} \\
=-\frac{1}{2 \beta}\left\{\operatorname { l n } \left[2 e^{9 / 4 \beta D_{A}} \cosh \left(\frac{3}{2} \beta \lambda_{A}\right)\right.\right. \\
\left.+2 e^{1 / 4 \beta D_{A}} \cosh \left(\frac{1}{2} \beta \lambda_{A}\right)\right] \\
+\ln \left[2 e^{25 / 4 \beta D_{B}} \cosh \left(\frac{5}{2} \beta \lambda_{B}\right)\right. \\
+2 e^{9 / 4 \beta D_{B}} \cosh \left(\frac{3}{2} \beta \lambda_{B}\right) \\
\left.\left.+2 e^{1 / 4 \beta D_{B}} \cosh \left(\frac{1}{2} \beta \lambda_{B}\right)\right]\right\} \\
+\frac{1}{2}\left(-z J m_{A} m_{B}+\lambda_{A} m_{A}+\lambda_{B} m_{B}\right),
\end{gathered}
$$

where $N$ is the total number of sites of lattice and $z$ its nearestneighbor coordination number. Minimizing this expression with respect to $\lambda_{A}$ and $\lambda_{B}$ gives self-consistent expressions for the mean field as follows:

$$
\lambda_{A}=z J m_{B}, \quad \lambda_{B}=z J m_{A},
$$

with

$$
\begin{aligned}
& m_{A} \equiv\left\langle\sigma_{i}^{A}\right\rangle_{o} \\
&= \frac{1}{2} \frac{3 \sinh \left((3 / 2) \beta \lambda_{A}\right)+e^{-2 \beta D_{A}} \sinh \left((1 / 2) \beta \lambda_{A}\right)}{\cosh \left((3 / 2) \beta \lambda_{A}\right)+e^{-2 \beta D_{A}} \cosh \left((1 / 2) \beta \lambda_{A}\right)} \\
& m_{B} \equiv\left\langle\sigma_{j}^{B}\right\rangle_{o} \\
&= \frac{1}{2}\left\{\left(5 \sinh \left(\frac{5}{2} \beta \lambda_{B}\right)+3 e^{-4 \beta D_{B}} \sinh \left(\frac{3}{2} \beta \lambda_{B}\right)\right.\right. \\
&\left.\quad+e^{-6 \beta D_{B}} \sinh \left(\frac{1}{2} \beta \lambda_{B}\right)\right) \\
& \quad \times\left(\cosh \left(\frac{5}{2} \beta \lambda_{B}\right)+e^{-4 \beta D_{B}} \cosh \left(\frac{3}{2} \beta \lambda_{B}\right)\right. \\
&\left.\left.\quad+e^{-6 \beta D_{B}} \cosh \left(\frac{1}{2} \beta \lambda_{B}\right)\right)^{-1}\right\}
\end{aligned}
$$

To evaluate the phase diagram (second-order phase transition lines) of a mixed-spin system, one has to expand (7), (9), that,

$$
g=g_{o}+a m_{A}^{2}+b m_{A}^{4}+\cdots
$$

where the coefficients $g_{o}$ and $a$ are given by

$$
\begin{aligned}
& g_{o}=-\frac{1}{2 \beta} \ln \left[\left(x_{A}+y_{A}\right)\left(x_{B}+y_{B}+z_{B}\right)\right], \\
& a=-\frac{1}{2 \beta}\left[\frac{t^{2}}{8} b_{2}+\frac{t^{4}}{128} a_{1} b_{1}^{2}\right]+0.125 t z J b_{1}
\end{aligned}
$$


with

$$
\begin{aligned}
& x_{A}=2 e^{9 / 4 \beta D_{A}}, \quad y_{A}=2 e^{1 / 4 \beta D_{A}}, \quad t=\beta z J, \\
& x_{B}=2 e^{25 / 4 \beta D_{B}}, \quad y_{B}=2 e^{9 / 4 \beta D_{B}}, \quad z_{B}=2 e^{1 / 4 \beta D_{B}}, \\
& z_{B_{1}}=e^{-4 \beta D_{B}}, \quad z_{B_{2}}=e^{-6 \beta D_{B}}, \quad b_{1}=\frac{25+9 z_{B_{1}}+z_{B_{2}}}{1+z_{B_{1}}+z_{B_{2}}}, \\
& b_{2}=\frac{25 x_{B}+9 y_{B}+z_{B}}{x_{B}+y_{B}+z_{B}}, \quad a_{1}=\frac{9 x_{A}+y_{A}}{x_{A}+y_{A}} .
\end{aligned}
$$

The second-order phase transition line is then determined by $a=0$ and $b>0$. It is worth mentioning that (12) could be evaluated both Ferromagnetic phase stability limit $(J>0)$ and Ferrimagnetic one $(J<0)[3,9,11]$.

On the other hand, the ferrimagnetic case shows that the signs of sublattice magnetizations are different, and there may be a compensation point, at which the total magnetization per site, that is,

$$
M=\frac{1}{2}\left(m_{A}+m_{B}\right)
$$

is equal to zero, but $\left(m_{A}=-m_{B} \neq 0\right)[3,12]$.

\section{Results and Discussions}

Firstly, the ground state of the system has been determined from the Hamiltonian (1), by comparing the energies of the corresponding configurations as shown in Figure 1. However, at zero temperature, one can find six phases with different values of $\left\{m_{A}, m_{B}, K_{A}, K_{B}\right\}$, namely, the Ferrimagnetic phases ordered as follows

$$
\begin{array}{ll}
O_{1} \equiv\left\{\frac{3}{2}, \frac{5}{2}, \frac{9}{4}, \frac{25}{4}\right\}, & O_{2} \equiv\left\{\frac{1}{2}, \frac{5}{2}, \frac{1}{4}, \frac{25}{4}\right\}, \\
O_{3} \equiv\left\{\frac{3}{2}, \frac{3}{2}, \frac{9}{4}, \frac{9}{4}\right\}, & O_{4} \equiv\left\{\frac{1}{2}, \frac{3}{2}, \frac{1}{4}, \frac{9}{4}\right\}, \\
O_{5} \equiv\left\{\frac{3}{2}, \frac{1}{2}, \frac{9}{4}, \frac{1}{4}\right\}, & O_{6} \equiv\left\{\frac{1}{2}, \frac{1}{2}, \frac{1}{4}, \frac{1}{4}\right\} .
\end{array}
$$

There are no disordered phases where the parameters $K_{A}$ and $K_{B}$, are defined by

$$
K_{A}=\left\langle\left(s_{i}^{A}\right)^{2}\right\rangle, \quad K_{B}=\left\langle\left(s_{j}^{B}\right)^{2}\right\rangle .
$$

Let us consider the case when $-6.0 \leq D_{B} /|J| \leq 1.0$. Figure 2 stands for the low-temperature phase diagram in the $\left(D_{B}, T\right)$ plane for the mixed-spin Ising ferrimagnet on a simple cubic lattice (coordination number $z=6$ ) using various values of $D_{A} /|J|$. Taking all ordered ferrimagnetic phases from the phase diagram under consideration, one can observe characteristic behaviours of second-order transitions at different values of $D_{A} /|J|$.

Figures 2 and 3 show, for different values of $D_{A}$ and $D_{B}$, the phase diagrams in the $\left(D_{B}, T\right),\left(D_{A}, T\right)$ planes, respectively. It has been observed, for both figures, that the ordered states exist at low temperatures for all values of anisotropies.

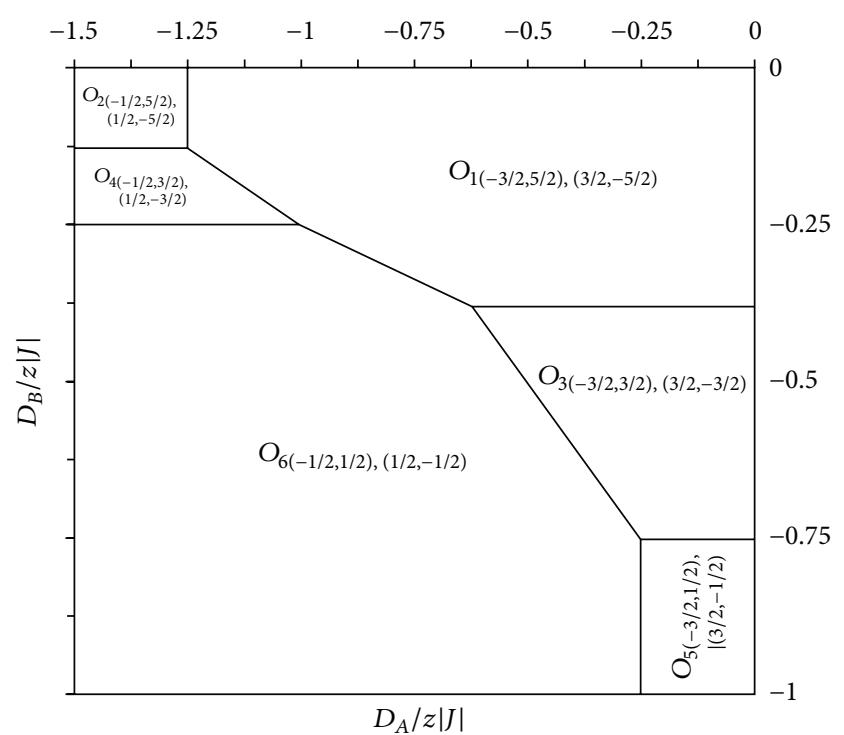

FIGURE 1: Ground-state phase diagram of the mixed spin-3/2 and spin-5/2 Ising ferrimagnetic system with the coordination number $z$ and different single-ion anisotropies $D_{A}$ and $D_{B}$. The six ordered phases $O_{1}, O_{2}, O_{3}, O_{4}, O_{5}$, and $O_{6}$ are separated by thin lines.

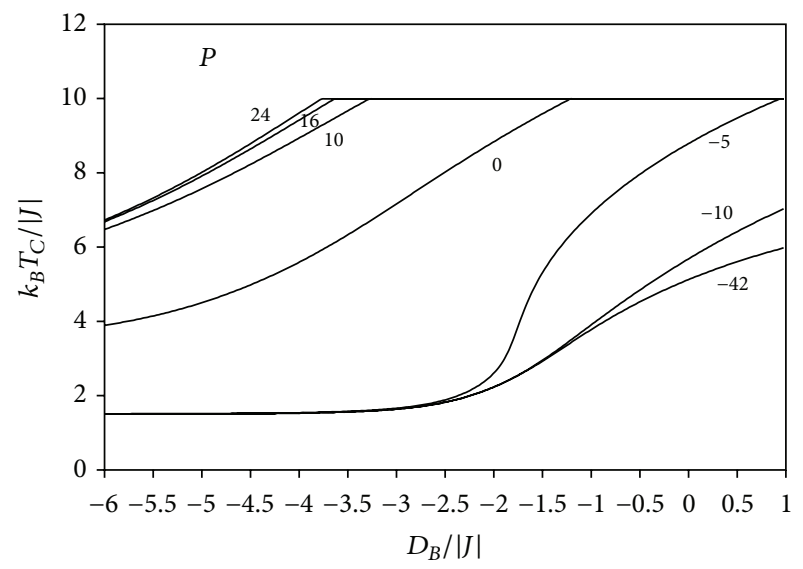

FIgURE 2: Phase diagram in the $\left(D_{B}, T\right)$ plane for the mixed-spin Ising ferrimagnet with the coordination number $z=6$, when the value of $D_{A}$ is changed. The solid lines indicate second-order phase transitions. $P$ is the paramagnetic phase.

So, one can obtain a transition temperature $\left(k_{B} T /|J|=\right.$ 11.4538) (see Figure 3), particularly, in the absence of anisotropies $\left(D_{A}=D_{B}=0.0\right)$. Moreover, one can see from Figure 2, at the values of $D_{A} /|J| \geq 24.0$, that the transition temperature can never be increased; for $D_{A} /|J| \leq-42.0$, the transition temperature can never be lowered.

Now, let us discuss the temperature dependence of the sublattice magnetizations $m_{A}$ and $m_{B}$, by solving the coupled (8)-(9) numerically.

As shown in Figure 4, when $D_{B} /|J| \leq-2.0$ for fixed $D_{A} /|J|=-4.0$, the sublattice magnetizations of mixture show characteristic thermal variation behaviour. On the other hand, within the range $-2.35 \leq D_{B} /|J|<-2.25$, the sublattice 


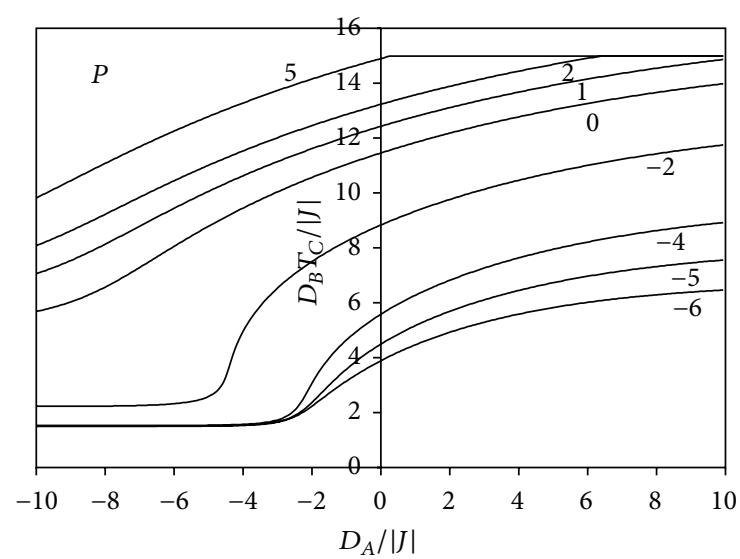

FIgURE 3: Phase diagram in the $\left(D_{A}, T\right)$ plane for the mixed-spin Ising ferrimagnet with the coordination number $z=6$, when the value of $D_{B}$ is changed. The solid lines indicate second-order phase transitions. $P$ is the paramagnetic phase.

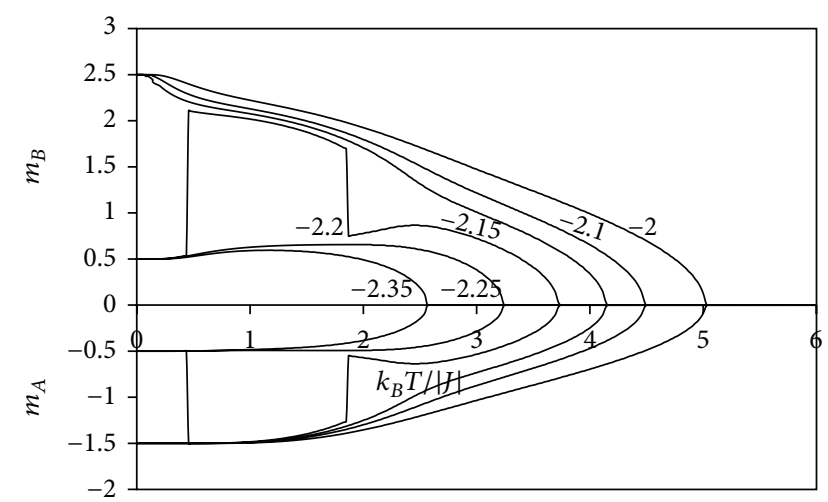

Figure 4: Thermal variations of the sublattice magnetizations $m_{A}$, $m_{B}$ for the mixed-spin Ising ferrimagnet with the coordination number $z=6$, when the value of $D_{B} /|J|$ is changed, for fixed $D_{A} /|J|=$ -4.0 .

magnetization $m_{A}$ shows normal thermal variation behavior (Q-type) [6].

As $D_{B}$ increases from $D_{B} /|J|=-2.25$ to -2.20 for a fixed value of $D_{A} /|J|$, the temperature dependence of $m_{A}$ may exhibit interesting features. So, it is observed that a sharp rapid increase from its saturation magnetization occurs. Figure 5 refers to the thermal variations of the sublattice magnetizations $m_{A}, m_{B}$ for the same system with the value $D_{B} /|J|$ varying for a fixed value of $D_{A} /|J|=-1.0$. For $-4.0 \leq$ $D_{B} /|J| \leq-4.4$, the temperature dependence of $m_{B}$ may exhibit a rather rapid decrease from its saturation value at $k_{B} T /|J|=0$. The phenomenon is further enhanced when the value of $D_{B} /|J|$ approaches the critical value. At the critical value of $D_{B} /|J|$, in particular, for $k_{B} T /|J|=0$, the saturation value of $m_{B}$ is 1.0 , indicating that in the ground state the spin configuration of the system consists of the mixed phase $s_{j}^{B}=$ $\pm 3 / 2$ or $s_{j}^{B}= \pm 1 / 2$ with equal probability. In other words, one should notice that a new behaviour, not predicted in the Neel

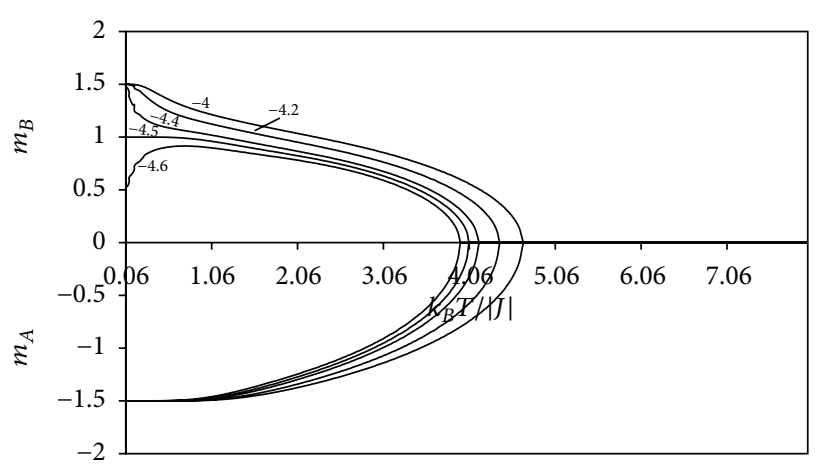

FIGURE 5: Thermal variations of the sublattice magnetization $m_{A}, m_{B}$ for the mixed-spin Ising ferrimagnet with the coordination number $z=6$, when the value of $D_{B} /|J|$ is changed, for fixed $D_{A} /|J|=-1.0$.

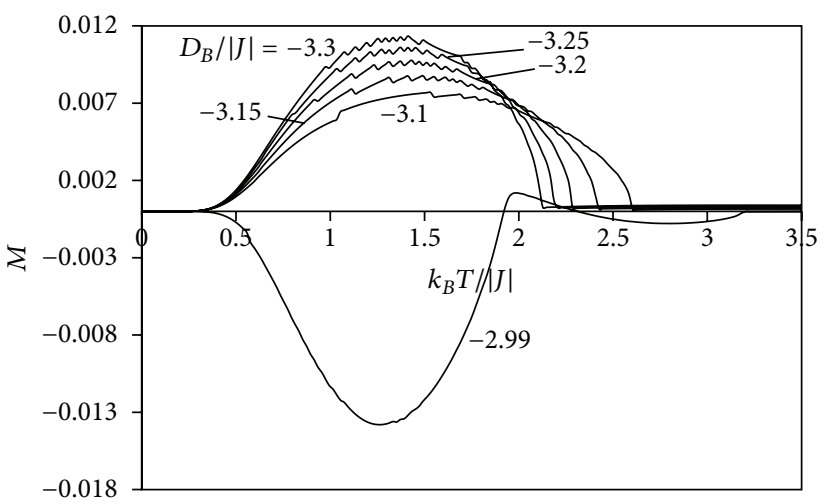

FIgURE 6: Thermal variations of the total magnetization $M$ for the mixed-spin Ising ferrimagnet with the coordination number $z=6$, when the value of $D_{B} /|J|$ is changed, for fixed $D_{A} /|J|=-2.90$.

theory of ferrimagnetism [6], is observed in our model, when a value of $D_{B} /|J|$ approaches the critical one $D_{B C} /|J|=-4.5$.

Now, it is interesting to examine the characteristic properties of the system in which we find the compensation temperature $\left(T_{k}\right)$ depends strongly on the values of anisotropies $D_{A}, D_{B}$ acting on the $A$-atoms and $B$-ones, respectively. As shown in Figure 6, the system may exhibit characteristic features in the temperature dependence of the magnetization depending on the values of anisotropies $D_{B}$ of the sites occupied by $B$-atoms for a particular value of $D_{A}$; that is, it is possible to have three compensation points at $k_{B} T /|J|=0$ and $k_{B} T /|J| \neq 0$, respectively.

In Figure 7, we report an interesting feature of compensation temperatures for selected values of anisotropies $D_{A} /|J|=$ $-2.90, D_{B} /|J|=-2.99$. As is seen from Figure 6, in the region where the system may show a compensation point, the sublattice magnetization $m_{A}$ is more ordered than the sublattice magnetization $m_{B}$ below compensation temperature. These sublattice magnetizations are still incomplete so there is a residual magnetization in the system. As the temperature is increased, at certain values of anisotropies $D_{A}, D_{B}$ acting on the $A$-atoms and $B$-atoms, respectively, the direction of this residual magnetization may switch. That is to say, due to entropy some spins can flip their directions. Thus, the 


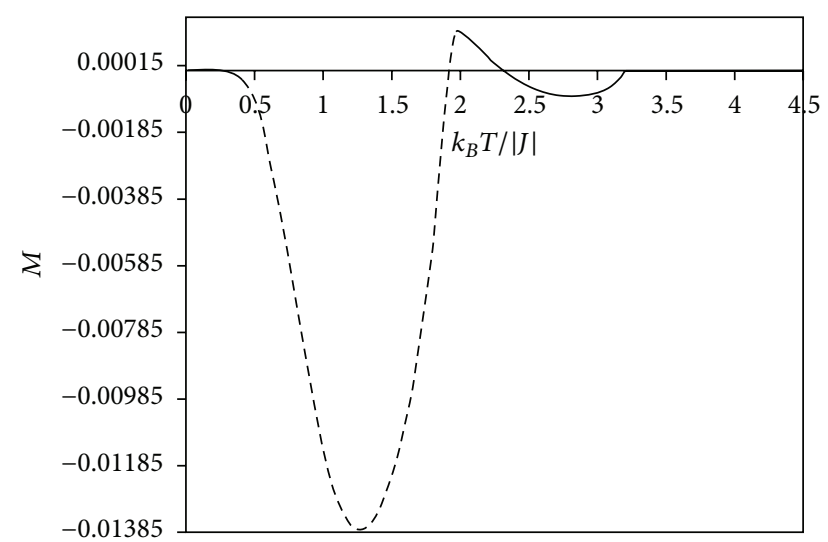

FIgURE 7: A close view of the temperature dependences of the magnetization for the mixed-spin Ising ferrimagnet with the coordination number $z=6$, when $D_{B} /|J|=-2.99$ and $D_{A} /|J|=-2.90$.

sublattice magnetization $m_{B}$ becomes more ordered than the sublattice magnetization $m_{A}$ for the temperatures above compensation one. So there is an intermediate point such that the cancellation is complete [10]. It is worth the noting that the mean-field theory for the ferrimagnetic case, predicts the existence of a compensation point in the ordered phase where the total moment vanishes $[3,7]$.

\section{Conclusions}

The mean-field approximation has been used in this treatment. The phase diagram of the mixed spin-3/2 and spin-5/2 Ising ferrimagnetic system with different single-ion anisotropies has been investigated. The magnetic properties of the system with different anisotropies have been found by solving the general expressions numerically. So, the magnetization curves have exhibited some characteristics; that is, it has been shown that the mixed-spin ferrimagnetic system, which is considered, may exhibit an outstanding feature (many compensation points). Thus, one can compare our results with these of a mixed spin- 1 and spin-3/2 system [3] and mixed spin-3/2 and spin-5/2 on the basis of the Bethe lattice [13], in which the mixed-spin model shows one or two compensation temperatures depending on the values of the crystal fields, respectively. It is worth the noting that our model may exhibit three compensation points. Furthermore, in a theoretical study dealing with the mixed spin-3/2 and spin-5/2 Ising model, which was treated using exact recursion relations on Cayley tree lattices [14], it has been found that the secondorder phase transition becomes sharper when anisotropies $D_{A}$ or $D_{B}$ acting on both atoms increase. One can compare with these behaviours investigated in our model for different values of both anisotropies, as shown in Figures 2 and 3 . We have observed that the decrease the anisotropy of an atom- $\mathrm{B}$ the decrease the transition temperature, as shown in Figures 4 and 5, whereas a transition temperature occurs at higher one for appropriate values of $D_{A} /|J|=-2.90$ and $D_{B} /|J|=-2.99$ (see Figure 6). The researchers [15], using the effective field theory for the mixed spin-3/2 and spin-5/2 Ising model on other regular planar lattices, have shown that the critical temperature becomes larger as the compensation temperature becomes smaller with the increase in the crystal field. These results seem to be in line with ours, when the value of $D_{B}$ is changed, for a fixed value of $D_{A}$, which one can observe Figures 6 and 7. However, as far as we are concerned whether a compensation point exists or not for such a system has not yet been proved, within the mean-field approximation based on the Bogoliubov inequality for the free energy. Finally, we hope that our present results may be helpful, for example, to support and clarify the characteristic features, for a simple cubic lattice, in a series of molecular-based magnets $\mathrm{Cs}_{2} \mathrm{Mn}^{\mathrm{II}}\left[\mathrm{V}^{\mathrm{II}}(\mathrm{CN})_{6}\right]$ [16], when the experimental data of ferrimagnetic materials are analyzed.

\section{References}

[1] W. Figueiredo, M. Godoy, and V. S. Leite, "Compensation temperature of the mixed-spin ising model on the hexagonal lattice," Brazilian Journal of Physics, vol. 34, no. 2A, pp. 392-394, 2004.

[2] E. Machado and G. M. Buendia, "Mixed Ising models with two compensation temperatures," Journal of Magnetism and Magnetic Materials, vol. 272-276, no. I, pp. 249-250, 2004.

[3] O. F. Abubrig, D. Horvath, A. Bobak, and M. Jascur, "Meanfield solution of the mixed spin-1 and spin- Ising system with different single-ion anisotropies," Physica A, vol. 296, pp. 437450, 2001.

[4] M. Jascur and J. Strecka, "Reentrant transitions of a mixed-spin Ising model on the diced lattice," Condensed Matter Physics, vol. 8, pp. 869-880, 2005.

[5] J. M. Yeomans, Ed., Statistical Mechanics of Phase Transitions, Oxford Science Publications, Oxford, UK, 1994.

[6] T. Kaneyoshi, M. Jascur, and P. Tomczak, "The ferrimagnetic mixed spin-1/2 and spin- 3/2 Ising system," Journal of Physics: Condensed Matter, vol. 4, no. 49, pp. L653-L658, 1992.

[7] J. S. da Cruz Filho, M. Godoy, and A. S. de Arruda, "Phase diagram of the mixed spin-2 and spin-5/2 Ising system with two different single-ion anisotropies," Condensed Matter, vol. 1, p. 13256, 2011.

[8] R. Ranganathan and A. Ray, "Ferrites: what is new?" PramanaJournal of Physics, vol. 58, no. 5-6, pp. 995-1002, 2002.

[9] A. Bobak, "The effect of anisotropies on the magnetic properties of a mixed spin-1 and spin-3/2 Ising ferrimagnetic system," Physica A, vol. 258, pp. 140-156, 1998.

[10] A. Dakhama and N. Benayad, "On the existence of compensation temperature in $2 \mathrm{~d}$ mixed-spin Ising ferrimagnets: an exactly solvable model," Journal of Magnetism and Magnetic Materials, vol. 213, pp. 117-125, 2000.

[11] K. Huang, Statistical Mechanics, McGraw-Hill, New York, NY, USA, 2nd edition, 1987.

[12] A. Bobak and D. Horvath, "Magnetic properties of diluted mixed spin-1/2 and spin-s Ising ferrimagnets with a crystal field," Physica Status Solidi B, vol. 213, no. 2, pp. 459-470, 1999.

[13] E. Albayrak and A. Yigit, "Mixed spin-3/2 and spin-5/2 Ising system on the Bethe lattice," Physics Letters A, vol. 353, no. 2-3, pp. 121-129, 2006.

[14] R. A. Yessoufou, S. H. Amoussa, and F. Hontinfinde, "Magnetic properties of the mixed spin-5/2 and spin-3/2 Blume-Capel Ising system on the two-fold Cayley tree," Central European Journal of Physics, vol. 7, no. 3, pp. 555-567, 2009. 
[15] Q. Zhang, G. Wei, and Y. Gu, "The study of the phase diagram and internal energy of the mixed spin-3/2 and spin-5/2 ferrimagnetic Ising system with interlayer coupling by effectivefield theory; a simple approach of calculating internal energy," Physica Status Solidi B, vol. 242, no. 4, pp. 924-932, 2005.

[16] W. R. Entley and G. S. Girolami, "High-temperature molecular magnets based on cyanovanadate building blocks: spontaneous magnetization at 230 K,' Science, vol. 268, no. 5209, pp. 397-400, 1995. 

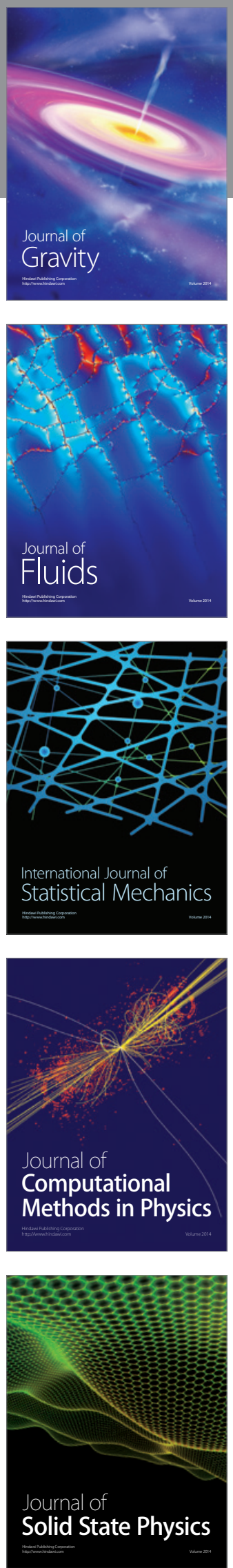

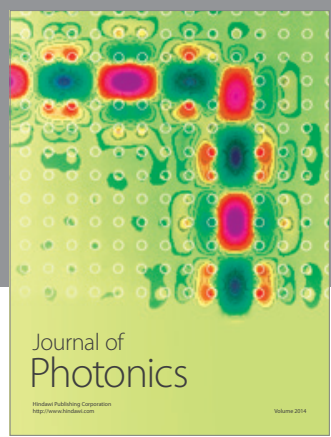

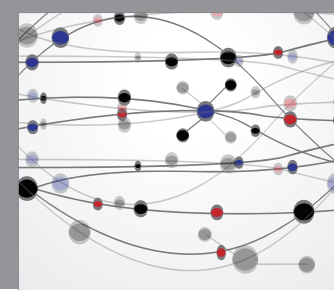

The Scientific World Journal

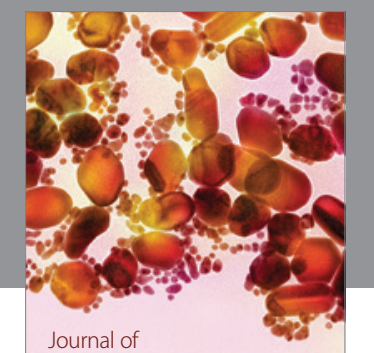

Soft Matter
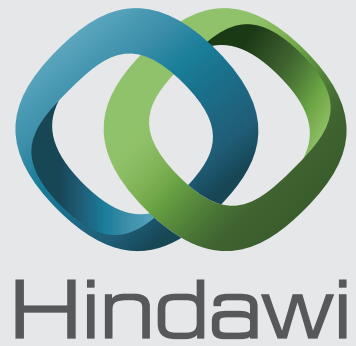

Submit your manuscripts at

http://www.hindawi.com
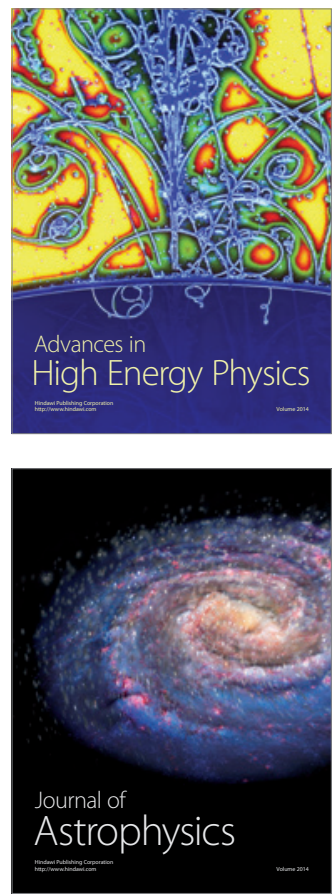
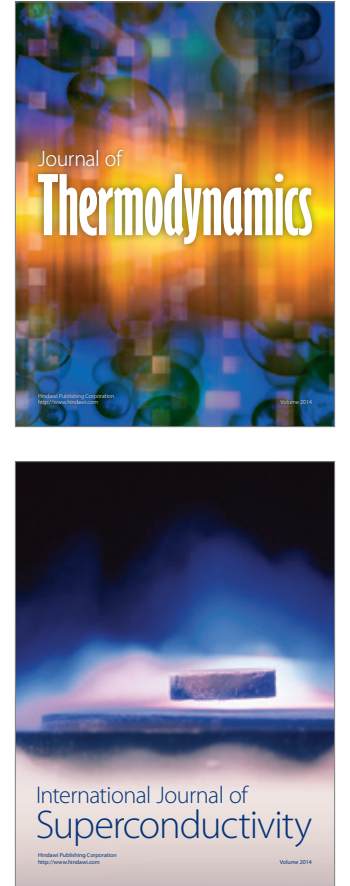
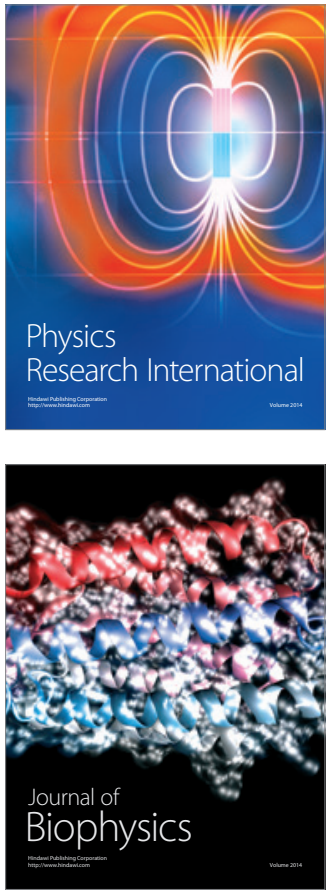
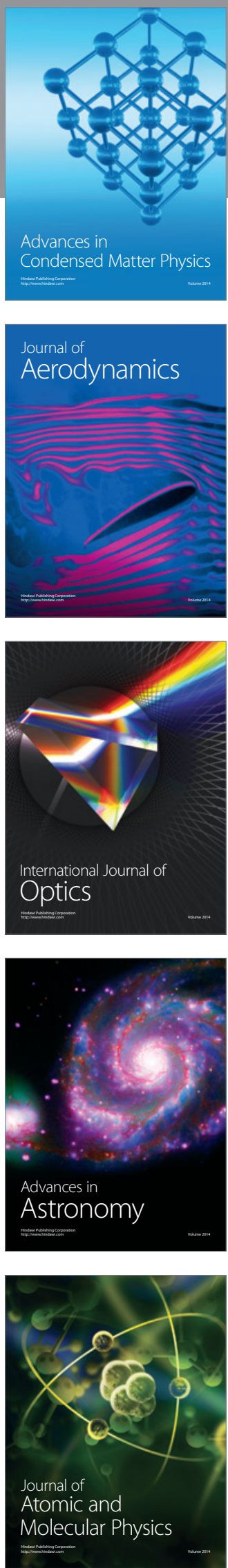